\title{
Facebook, medio de comunicación de las organizaciones campesinas del Catatumbo*
}

\author{
J. F. Barbosa Trigos ** \\ E. F. Ríos Pacheco ${ }^{* * *}$ \\ Recibido: 2021-02-14 • Enviado a pares: 2021-02-20 \\ Aprobado por pares: 2021-03-21 • Aceptado: 2021-04-19 \\ https://doi.org/10.22395/angr.v20n39a8
}

\begin{abstract}
Resumen
El artículo responde a la investigación titulada Evolución e impacto de la red social Facebook como medio de comunicación masiva de las organizaciones campesinas del Catatumbo, Ascamcat y Cisca, desarrollada para identificar las dinámicas existentes en el uso del medio social como una herramienta de difusión de las actividades propias de las organizaciones citadas, teniendo en cuenta la importancia de sus acciones mediáticas en la subregión del departamento Norte de Santander, el Catatumbo colombiano, de allí la relevancia para los habitantes del lugar, quienes pueden aprovechar la herramienta en su progreso social.

La investigación recolectó datos cualitativos por medio de observación y entrevistas semiestructuradas, dando lugar a la comprensión que la Web 2.0 trajo, y así determinar las dinámicas producto del uso de Facebook como medio de comunicación por parte de las organizaciones campesinas del Catatumbo, Ascamcat y Cisca.

Para la Asociación Campesina del Catatumbo y el Comité de integración Social del Catatumbo, Facebook es una herramienta que apoya tres ejes: visibiliza las actividades desarrolladas por cada organización; muestra las realidades propias de la región integrada por once municipios en el departamento Norte de Santander; y desmiente o contrarresta informaciones emitidas por los medios de comunicación masiva del orden departamental y nacional.
\end{abstract}

Por consiguiente, aunque la red social virtual no fue creada ni diseñada para ser un medio de comunicación masiva, en esencia se convierte en la ventana que permite mostrar las realidades del Catatumbo, desmintiendo en algunos casos el discurso hegemónico de los tradicionales medios, radio, prensa y televisión.

Palabras clave: organización, clase campesina, red social, medios sociales, medios de comunicación de masas, tecnología de la comunicación y zona rural.

\footnotetext{
El texto se desprende de la investigación titulada Evolución e impacto de la red social Facebook como medio de comunicación masiva de las organizaciones campesinas del Catatumbo, Ascamcat y Cisca, financiada por la Universidad Francisco de Paula Santander Ocaña y realizada en la región del Catatumbo colombiano, zona norte del departamento Norte de Santander. Colombia.

“ Docente investigador del Grupo de Investigación Communis de la Universidad Francisco de Paula Santander Ocaña, Colombia. Comunicador Social, Magíster en Comunicación Digital de la Universidad Pontificia Bolivariana. Orcid: https://orcid.org/0000-00023011-0441. Correo electrónico: jfbarbost@ufpso.edu.co

... Docente investigador del Grupo de Investigación Communis de la Universidad Francisco de Paula Santander Ocaña, Colombia. Comunicador Social, Magíster en Desarrollo Local del Isead (España) y la Universidad Francisco de Paula Santander Cúcuta. Orcid: https://orcid.org/0000-0001-8983-4156. Correo electrónico: efrios@ufpso.edu.co
} 


\title{
Facebook as a Communication Media in Farmers' Organizations in Catatumbo
}

\begin{abstract}
This article is an answer to the research titled as Evolution and impact of the Facebook social network as mass media for the Catatumbo farmers' organizations Ascamcat and Cisca, developed for identifying the dynamics between the use of social media as a tool for broadcasting the activities of these groups, taking into account the importance of their media actions in the subregion of the North of Santander region, the Colombian Catatumbo, therefore its relevance for their inhabitants, who can take profit of this tool for their social progress.

The research gathered qualitative data through observations and semi-structured interviews, giving place to the comprehension given by the $2.0 \mathrm{Web}$, and thus determining the dynamics that are a byproduct of the use of Facebook as a communication media by farmer's organizations of the Catatumbo region, Ascamcat and Cisca. For the Catatumbo's Farmers' Association and Social Integration Committee of Catatumbo, Facebook is a tool that works in three axes: making visible the activities developed by each organization; displaying the realities of each region, integrated by 11 municipalities in North of Santander and redressing the information issued by the national and regional mass media

Therefore, even though the social network was not created or designed for becoming a mass media, it is, essentially, a window for displaying the realities of the Catatumbo region, redressing in some cases the hegemonic discourse of the traditional media: radio, press and television.
\end{abstract}

Keywords: organization, farmer class, social network, social media, mass media, communication technologies, rural area.

\section{Facebook, meio de comunicação das organizações rurais de Catatumbo}

\begin{abstract}
Resumo
O artigo da resposta a investigação titulada Evolución e impacto de la red social Facebook como medio de comunicación masiva de las organizaciones campesinas del Catatumbo, Ascamcat y Cisca, desenvolvida para identificar as dinâmicas existentes no uso do meio social como um instrumento de divulgação das atividades próprias das organizações referidas, tendo em conta a importância das suas ações midiáticas na sub-região do estado Norte de Santander, no Catatumbo colombiano, daí a relevância para os habitantes do lugar, quem podem aproveitar o instrumento em seu progresso social.

A investigação coletou dados quantitativos através de observação e entrevistas semiestruturadas, resultando a compreensão que a Web 2.0 trouxe, e assim determinar as dinâmicas, fruto do uso do Facebook como meio de comunicação por parte das organizações rurais de Catatumbo, Ascamcat e Cisca.

Para a Associação Rural do Catatumbo e o Comité de integração Social do Catatumbo, o Facebook é um recurso que apoia três eixos: visualização das atividades desenvolvidas por cada organização; mostra as realidades próprias da região composta por onze municípios no estado Norte de Santander; e desmente e anula informações emitidas pelos meios de comunicação massivo estadual e nacional.

Consequentemente, mesmo que que a rede social não foi criada nem projetada para ser um meio de comunicação massiva, basicamente se converte na janela que permite mostrar as realidades do Catatumbo, desmentindo em algumas situações o discurso hegemônico dos tradicionais veículos: rádio, imprensa e televisão.

Palavras chave: organização, classe rural, rede social, meios sociais, meios de comunicação de massas, tecnologia da comunicação e zona rural.
\end{abstract}




\section{Introducción}

La subregión del Catatumbo colombiano, ubicada en el departamento Norte de Santander, integra once municipios (Ocaña, Ábrego, Sardinata, Convención, Teorama, Hacarí, San Calixto, Tibú, El Tarra, El Carmen y La Playa de Belén). Siendo una región de carácter agrícola y comercial, su mayor extensión se encuentra en la zona rural, lugar donde existen cooperativas, asociaciones de productores, organizaciones campesinas y grupos insurgentes, como actores de la región.

El desarrollo de las Tecnologías de la Información y las Comunicaciones (TIC) en el departamento, al igual que en el resto del país, es una apuesta del Gobierno Nacional que procura aumentar la cobertura tecnológica y la conectividad. Sin embargo, dicha visión no está completamente implementada en el Catatumbo, región que durante años ha sido olvidada por el Estado colombiano y asediada por la presencia de grupos insurgentes que se disputan permanentemente el territorio.

No obstante, en indudable que las TIC benefician las acciones de líderes que trabajan por el bienestar de sus comunidades. Investigaciones previas demuestran que los medios sociales como Facebook, permiten generar información propia de diferentes grupos poblacionales (asociados o no), brindando a los usuarios (seguidores o no), información diferente a la transmitida por los medios de comunicación masiva tradicionales.

La comunicación de masas promovida por los llamados medios de comunicación de masas, siendo básicamente de información, durante la sociedad de la información y el conocimiento se transforma. En palabras de Castells (2009), la nueva autocomunicación de masas refleja la realidad en red en la cual vivimos las personas (conectadas o no) una sociedad en la que cada individuo es un nodo, un punto latente de información y de libertad de expresión.

Sin embargo, en la sociedad actual donde se evidencia la innovación de tecnología, caracterizada por el "avance de la digitalización, de la transmisión y almacenamiento de textos, de datos, imágenes animadas; generalización de equipamientos cada vez más potentes (...) desarrollo fulminante de usos variados; internet e internet de alta velocidad" (Paquienséguy, 2007, p. 4), es palpable el cambio del linaje analógico hacia el linaje numérico; las nuevas TIC identificadas por "su total digitalización, por la posibilidad de ingresar a una red o a una conexión (a una red amplia y abierta o bien a otro aparato) y a menudo por una miniaturización de las herramientas volviéndose terminales de bolsillo" (p. 5), presentan fallas, no técnicas por su puesto, sino relacionadas con su uso y apropiación. Así, dice Simón (2016) que:

El cambio digital, es decir, la digitalización de la información, la generalización del protocolo de internet y la rápida adopción de estas 
tecnologías (equipos de usuario final, infraestructuras de banda ancha, etc.), está transformando la forma en la que producimos, almacenamos, distribuimos y consumimos bienes y servicios. (p. 1)

En este contexto, comprender el capital social como algo invisible, es decir, creado en la medida en que se establecen relaciones personales y colectivas, bien para desarrollar una actividad o simplemente para fortalecer lazos de confianza y amistad, es otro punto insoslayable, máxime cuando hablamos más que de culturas, de ciberculturas, nuevas comunidades, particularmente virtuales.

En pocas palabras, el hecho de relacionarte con alguien bajo principios de calidad más que de cantidad, con fines o intereses comunes, forma un capital social, que en últimas construye y, en estos momentos urgentemente, requiere reconstruir el tejido social y el entramado de redes que lo soportan; ejemplo de ello son las dinámicas conducentes a verdaderos progresos como el de las comunidades indígenas de México donde "poseen una estructura reticular que las lleva a avanzar de manera natural y ascendente hacia la generación y distribución de información estratégica a través de internet a fin de generar conocimiento" (Gómez, 2015 p. 2); lo expuesto genera un desarrollo a escala humana, como bien define Manfred Max-Neef (1993):

Tal desarrollo se concentra y sustenta en la satisfacción de las necesidades humanas fundamentales, en la generación de niveles crecientes de autodependencia y en la articulación orgánica de los seres humanos con la naturaleza y la tecnología, de los procesos globales con los comportamientos locales, de lo personal con lo social, de la planificación con la autonomía y de la sociedad civil con el Estado. (p. 30)

La libertad personal, y aún más, colectiva, capaz de transformar realidades, está caracterizada por dos elementos: ideas y trabajo. En ese sentido, dice Castells (2009) que, "[e]s la capacidad relacional de un actor social de influir de forma simétrica en las decisiones de otros actores sociales de modo que se favorezcan la voluntad, los intereses y los valores del actor" (p. 3).

Las nuevas formas de comunicación privilegiadas por internet, masifican el uso de esa libertad, particularmente relacionada con el uso de la información. Hoy adquieren especial relevancia los medios alternativos y el pueblo en general, quienes con mayor libertad pueden incidir en el pensamiento colectivo visibilizando comunidades habitualmente excluidas.

Las redes sociales y bitácoras personales, son hoy por hoy los espacios de convergencia virtual de culturas, de realidades personales y colectivas, dispuestos para construir nuevas formas de relación en la realidad virtual. Así, vemos diferentes espacios donde las personas se unen entorno a gustos musicales, intereses comunes, pertenencia a una ciudad, proyectos de vida, entre otras pretensiones. 
Algunos son espacios utilizados para dar visibilidad a las minorías, llevando lo local y/o regional hacia lo mundial, como sucede en la organización de ciberturbas, consideradas estas como "la culminación en la calle de un proceso de discusión social, llevado a cabo por medios electrónicos de comunicación y publicaciones personales en el que se rompe la división entre ciberactivistas y movilizados" (De Ugarte, 2007, p. 12).

El aprovechamiento de las TIC, recordando el derecho a la libertad de expresión, es entendido como un camino que propicia los liderazgos en comunidades apartadas de la geografía nacional, oscuras para la agenda gubernamental. Parafraseando a De Pablos (1999), en la revolucionaria era de la información se presenta la desaparición de oscuridades informativas. A mayor número de datos revelados, mayor iluminación informativa, menor oscuridad.

La transparencia, la voluntad política, la educación y la participación en entornos virtuales, son elementos imprescindibles en la relación democracia y sociedad. Lo anterior, dado que en la democracia se establecen relaciones entre los actores vinculados, donde la interpretación juega un papel preponderante porque procura la aplicabilidad de la libertad más acertada posible, debido a la diversidad de intereses, principios, valores o creencias de la sociedad.

Es allí donde el conocimiento rompe las tradicionales relaciones personales. El antiguo mecanismo de dominio de quienes ostentaban el conocimiento hoy, es cambiado por la libre circulación de la información. Surge aquí una pregunta cla sociedad ejerce asertivamente su libertad natural hoy apoyada en los nuevos medios tecnológicos?, recordando que las comunidades "son estructuras sociales contradictorias surgidas de conflictos y negociaciones entre diversos actores sociales a menudo opuestos" (Castells, 2009, p. 38).

Al respecto, las redes son fundamentales para el normal funcionamiento social, es indispensable el trabajo en red para lograr objetivos comunes y particulares, siempre trabajando como un engranaje donde cada pieza aporta un pequeño esfuerzo.

Si internet rompe las fronteras tradicionalmente conocidas, es importante comprender que crea en las dinámicas de la red nuevas fronteras ligadas al carácter discursivo y cultural, es decir, existen exclusiones por causa de gustos o la diversidad del consumo informativo.

Actualmente podríamos hablar de ecclesias digitales, siendo internet la herramienta que propicia sin limitantes territoriales el debate, los consensos y disensos, incidiendo en los temas de la vida pública. En este sentido, dice Castells (2009) que, "[i]nternet garantiza la interactividad en las reuniones de la ecclesia virtual (...) La ciudadanía puede reunirse cuando quiera y adoptar las decisiones que estime pertinentes en tiempos brevísimos, en decisiones instantáneas. Vuelve la democracia directa" (p. 10). 
El enorme potencial de comunicación de las redes telemáticas, radica no solo en el aumento de la capacidad de procesamiento de la información, sino también en la posibilidad de establecer comunicaciones horizontales no jerarquizadas. La creación de comunidad a través del uso de las redes telemáticas puede llevar a la generación del capital social tan anhelado por politólogos y políticos norteamericanos (Harto de Vera, 2006).

Las realidades física y virtual son esferas de la vida. Si es posible construir nación física al unirnos con otras personas compartiendo pensamientos, ideas, tradiciones, entre otros, es posible construir sociedad en la virtualidad, donde expandimos horizontes y se agrupan las personas por intereses comunes, aunque distantes físicamente.

La viralidad de la información en internet, surge por la existencia de redes pequeñas, muchas veces con límites difusos que generan la unión a otras redes más grandes, donde pueden generarse afinidades y liderazgos capaces de convencer a otros con ideas, aceptadas con conocimiento o no del tema. En este sentido, dice Castells (2009) que:

Las decisiones de las personas parecen basarse, sobre todo en ciertos períodos históricos, mucho más en la imitación o en la consideración de lo que los vecinos hacen que en la evaluación más o menos objetiva de las consecuencias de una conducta determinada. Tanto en las redes de la realidad física como en la virtual, los nodos que adquieren mayor fuerza son aquello más interconectados a otros, tienen mayor credibilidad y fuerza, que aquellos donde su red está integrada por pocas conexiones. Así "la sociedad red es una sociedad global. Ello no significa que todo el mundo participe de las redes (...) Pero todo el mundo se ve afectado por los procesos que tienen lugar en las redes globales. (p. 51)

Será fundamental retomar la importancia del ciudadano, de las minorías y de las mayorías, quienes utilizando deliberada, justa y sanamente la tecnología para el beneficio podrá cambiar las realidades actuales.

Partiendo de lo expuesto, surge la necesidad de determinar las dinámicas producto del uso de Facebook como medio de comunicación por parte de las organizaciones campesinas del Catatumbo, Ascamcat y Cisca.

\section{Metodología}

La presente investigación se desarrolló bajo la metodología cualitativa. La investigación profundizó en el conocimiento de una realidad particular, el uso que dos asociaciones de campesinos en el Catatumbo colombiano dan a Facebook. 
Su alcance fue exploratorio, porque pretendió conocer una realidad poco investigada, cumpliendo su finalidad, según Hernández-Sampieri et al. (2014), familiarizarnos con fenómenos relativamente desconocidos o novedosos, conforme a los resultados que arroja la revisión de la literatura.

Además, identificó las características sin establecer sus causas, describiendo el fenómeno objeto de estudio para detallar cómo y en qué circunstancias se presenta, lo anterior partiendo de una característica del estudio cualitativo, según HernándezSampieri et al. (2014), la comprensión de los fenómenos, explorándolos desde la perspectiva de los participantes en un ambiente natural y en relación con su contexto.

En el estudio se comprendieron las dinámicas producto del uso de Facebook como medio de comunicación por parte de las organizaciones campesinas del Catatumbo, Ascamcat y Cisca, estableciendo además los tipos y tratamientos de la información publicada en la red social virtual.

Teniendo en cuenta la naturaleza del estudio, el muestreo utilizado fue cualitativo, se escoge por criterios los casos de estudio, es decir, "elección de unidades porque representan o cubren uno o más criterios" (Hernández-Sampieri, et ál., 2014, p. 16), por lo anterior se definieron los siguientes criterios: organización social de carácter campesino, organización fundada y asentada en la región del Catatumbo, Norte de Santander y organización que tenga cómo mínimo cuenta o perfil en la red social Facebook.

Según Mejía (2016), en la subregión del Catatumbo "tres movimientos campesinos han tenido en los últimos años una gran influencia en las decisiones que se toman sobre esta convulsionada región del nororiente del país" (párr. 3). Las organizaciones son la Asociación Campesina del Catatumbo (Ascamcat), El Comité de Integración Social del Catatumbo (Cisca) y El Movimiento por la Constituyente Popular (MCP); de estas fueron escogidas las dos primeras, quienes respondieron a los tres criterios definidos anteriormente.

De las dos seleccionadas en la muestra, se vincularon los integrantes de las juntas directivas de cada organización o, en su defecto, quienes estaban encargados de las comunicaciones de la misma, ya que son quienes conocen y adelantan el proceso informativo en las redes sociales.

Inicialmente se realizaron entrevistas semiestructuradas; seguidamente se hizo observación minuciosa durante un mes de la red social usada por Ascamcat y Cisca, en donde se identificó tipo de información, periodicidad y sus características, formatos utilizados, posible público o públicos a quienes van dirigidos, entre otros aspectos. 


\section{Resultados}

Las organizaciones campesinas del Catatumbo, Asociación Campesina del Catatumbo (Ascamcat) y Comité de Integración Social del Catatumbo (Cisca), como su nombre lo menciona, son organizaciones de base campesina que lideran procesos de cambio social en la región donde trabajan, que tienen como meta principal el reconocimiento del campesinado colombiano como sujeto político y de derecho. El Cisca presenta un factor diferenciador y es su filiación al movimiento comunal y cooperativo.

Ambas nacen de la lucha por la reivindicación de los derechos vulnerados producto del conflicto interno colombiano y por el abandono estatal que la región del Catatumbo en Norte de Santander ha sufrido por más de cincuenta años.

Organizativamente, mientras Ascamcat está legalmente constituida con personería jurídica, integrando trece carteras (Comunicación, Sustitución, Educación, Organización, Agropecuaria, Salud, Mujeres, Relaciones políticas, Proyectos productivos, Paz e implementación, Administración, Gestión y finanzas, y Defensa del territorio); el Cisca es una organización de hecho sin personería jurídica, la cual basa su organización en las juntas de acción comunal, las asociaciones de juntas y el cooperativismo, fundamentando su accionar en tres principios: la integración, la vida y el territorio.

Las organizaciones campesinas del Catatumbo, Ascamcat y Cisca, son organizaciones caracterizadas por la lucha comunitaria para el logro de objetivos comunes, en ese sentido, "son agrupaciones de base, formales o informales, voluntarias, democráticas, cuyo fin primario es promover los objetivos económicos o sociales de sus miembros" (FAO, 1994, párr. 1). Así en palabras de la Organización de las Naciones Unidas para la Alimentación y la Agricultura (FAO) (1994), la situación jurídica no es lo importante, porque su fin radica en la existencia de un objetivo común; para el caso particular, mientras la Asociación Campesina del Catatumbo tiene personería jurídica, el Comité de Integración Social del Catatumbo es una organización de hecho sin personería jurídica fundamentada en el movimiento comunal y cooperativo de la región, sin existencia visible de junta directiva o representación legal.

La relación con los medios sociales, específicamente con el medio social Facebook, inició entre 2016 y 2017, cuando decidieron abrir Fan Pageslo que permitió cumplir tres objetivos fundamentales: 1. Mostrar y legitimar las actividades realizadas por cada organización; 2. visibilizar las realidades y hechos no precisamente violentos de la región; y 3. difundir informaciones que contrarrestan los discursos de los medios de comunicación masiva de carácter departamental y nacional.

Iniciar el acercamiento al citado medio social, también se debió a la necesidad de interactuar con personas del ámbito regional, nacional e internacional. Por lo anterior, para Ascamcat y Cisca los seguidores inicialmente fueron pocos, sus mismos 
miembros, tema que aumentó vertiginosamente sobre todo en momentos de mayor tensión en la región como se muestra en la figura 1 y figura 2.

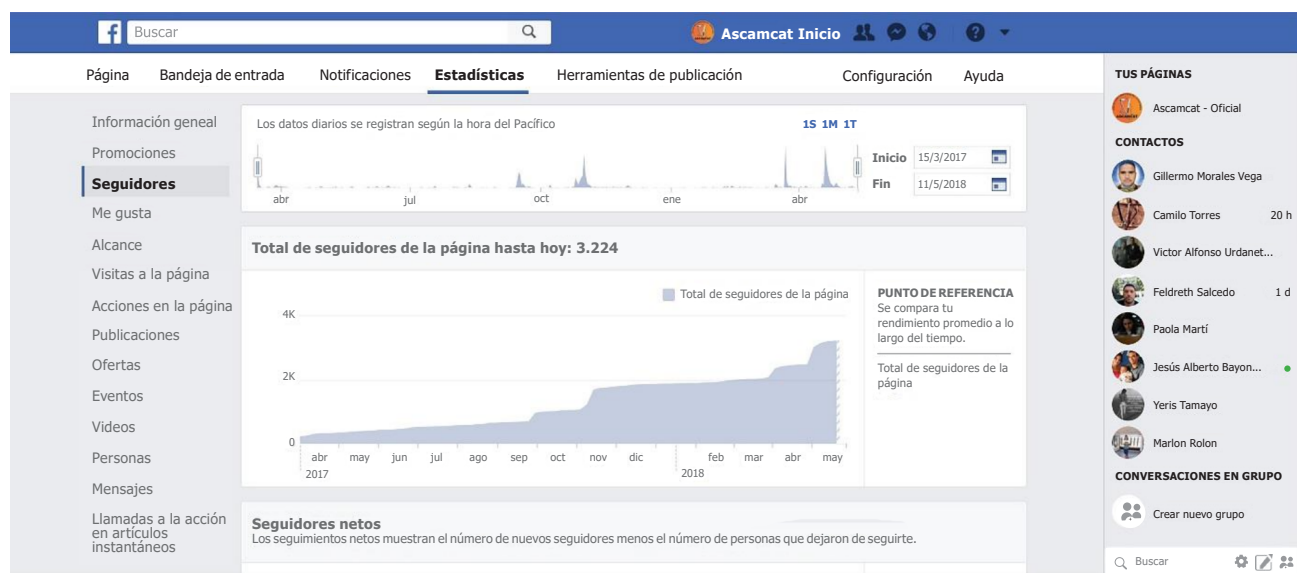

Figura 1. Seguidores generales de la página de Ascamcat

Fuente: tomada de la Fan Page de Ascamcat, 2018.

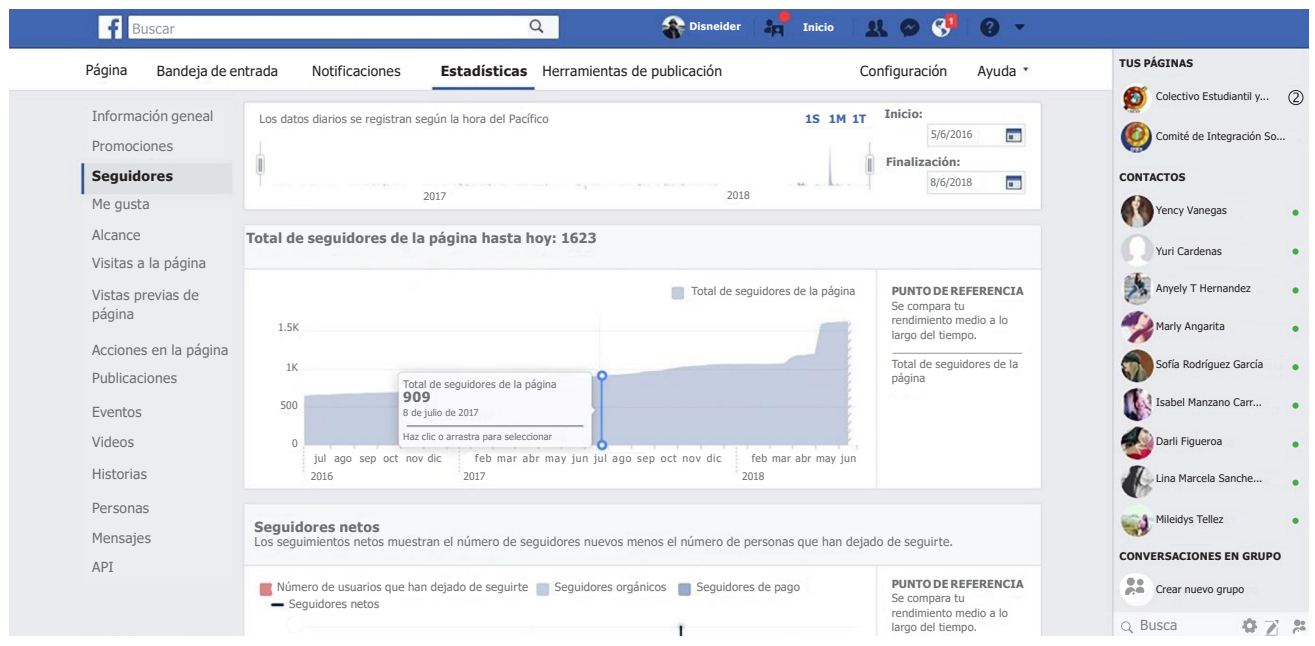

Figura 2. Seguidores generales de la página del Cisca

Fuente: Tomada del perfil personal de un integrante del Cisca, 2018.

El caso del primer acercamiento del Cisca a la red social virtual, integró dificultades culturales por causa del desconocimiento de estas herramientas en el Catatumbo, zona donde por su difícil topografía la conectividad a internet no es óptima y además es intermitente. La región, según Colombia TIC (2018), solo tiene cuarenta y dos Puntos Vive Digital, instalados para la educación digital de la población. 
Otra dificultad similar al iniciar el uso de la red social Facebook por ser organizaciones de base campesina, fue la falta de formación y competencias digitales necesarias para su manejo, lo cual impedía la publicación constante de entradas y la generación de contenido propio; por ello antes de usar Facebook tenían alianzas con medios alternativos como Periódico Periferia, Colombia Informa y Agencia Prensa Rural, donde publicaban sus informaciones.

Los contenidos publicados en las organizaciones son completamente públicos e integran diversos formatos como el texto, video y fotografía (siendo el texto el más usado). Los contenidos publicados suelen ser de temas como derechos humanos, memoria histórica, conflicto, situaciones de la región, movilizaciones, encuentros, y reuniones, publicados en forma de comunicados y noticias.

Cada organización tiene un equipo de comunicación integrado en promedio por cinco personas, quienes con formación profesional o no realizan el proceso de reportería, procesamiento de la información y posterior difusión, existiendo una revisión previa por parte de los líderes del proceso. Toda información cumple tres parámetros, veracidad de la información, existencia real de la fuente y contrastación de la información.

Algunas publicaciones son contenido propio y la mayoría son contenido compartido, contenido generado en otras plataformas y organizaciones que simplemente se coloca en la página oficial de la organización en Facebook, aclarando que las del primer tipo son las que logran mayor interacción con los usuarios. A continuación, se muestra en la figura 3 y figura 4 las características de los contenidos difundidos durante la observación realizada.

Las figuras permiten evidenciar que aumenta el número de publicaciones, aunque no es alto el número de las publicaciones, dado el número de veces compartida, tema que amplía el alcance de la misma. Lo anterior, en la medida en que una publicación puede ser observada por más usuarios sin que estos sean seguidores del Fan Page.

Además, es importante evidenciar que normalmente las organizaciones campesinas analizadas no generan contenido propio en su plataforma virtual, normalmente el contenido que es generado por otros usuarios de Facebook, Ascamcat y Cisca solo es compartido, de acuerdo con los intereses y la razón de ser de cada organización.

Al respecto, este tema se modifica un poco en los momentos de tensión en la región, es decir, cuando existen paros armados, paros campesinos u otro tipo de situación que altere el orden público. En estas circunstancias cada organización se encarga de mantener informados a sus usuarios sobre el acontecer de un hecho 


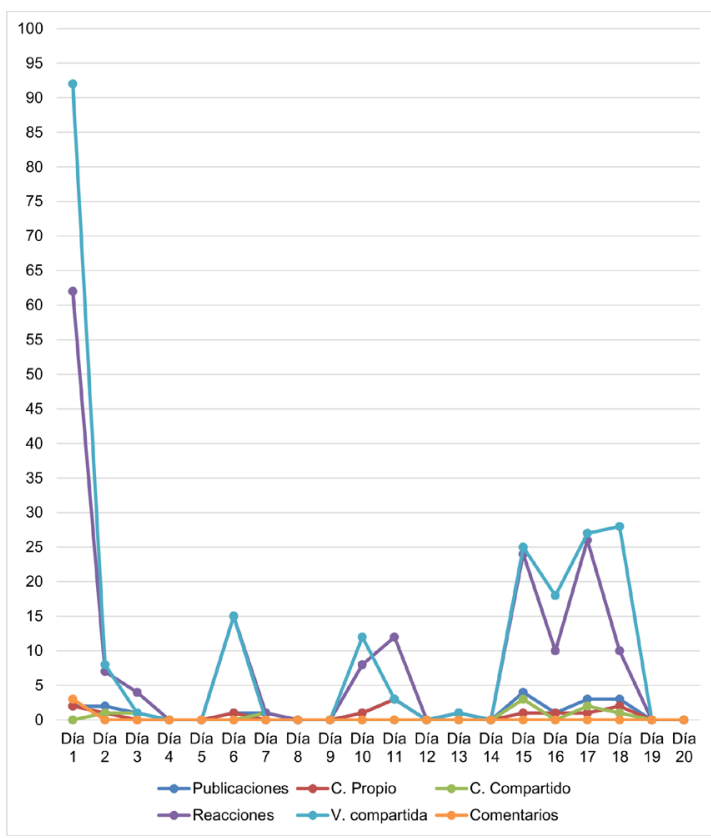

Figura 3. Características de las publicaciones del Cisca Fuente: elaboración propia.

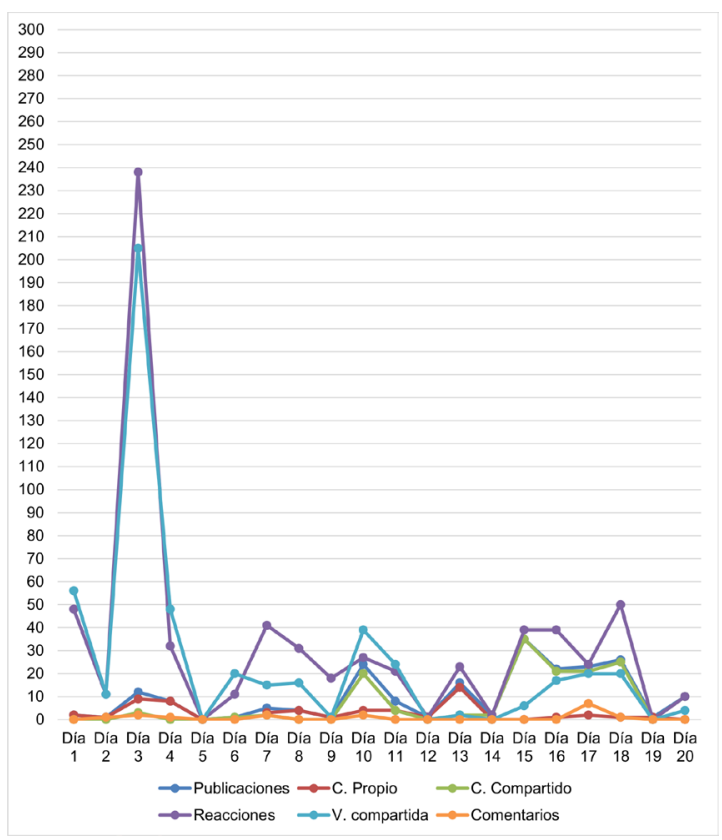

Figura 4. Características de las publicaciones de Ascamcat Fuente: elaboración propia. 
particular como los mencionados; en este punto los Fan Pages de cada organización sí logran convertirse en medios de comunicación expeditos que muestran la realidad de lo que viven, en contraposición a lo mostrado por los medios masivos de comunicación tradicionales.

A pesar de que Facebook es un medio que promueve la interacción entre los usuarios, no es recurrente. Lo anterior, debido a que se da especialmente en momentos de convulsión como los expuestos, tema que no es permanente en la región.

En ambas organizaciones analizadas, existe una política clara: no se responden a los comentarios hechos por los usuarios, esto porque no crearon el espacio para generar debate y porque por temas de seguridad se mantienen al margen de las discusiones. El espacio solo privilegia la emisión de información.

Frente al uso de Facebook, ambas organizaciones evidencian como principal ventaja la posibilidad de visibilizar sus actividades, así como las realidades, situaciones o hechos que acontecen en la región del Catatumbo, mostrarlas al interior y fuera de ella. Sin embargo, la visibilidad se da especialmente en contextos nacionales e internacionales, debido a las deficiencias presentes en la región, "conectividad, accesibilidad y comunicabilidad" (Duarte y Pires, 2011, p. 13), esta última entendida como el uso libre de las TIC y su apropiación sin ningún tipo de obstáculo.

Los integrantes de los equipos de comunicaciones, encargados de la difusión de los contenidos en Facebook, suelen ser residentes de la zona rural con poca o nula formación digital. No obstante, tres características importantes marcan la evolución del uso específicamente de Facebook en Ascamcat y el Cisca: en primer lugar, la vinculación de jóvenes en el proceso; en segundo lugar, la vinculación de personal con conocimiento o formación en el tema; y en tercer lugar, la permanente formación autodidacta en el manejo de la herramienta.

La Asociación Campesina del Catatumbo y El Comité de Integración Social del Catatumbo, presentan un patrón de difusión sin frecuencia establecida, podría decirse que se publican contenidos acorde a las necesidades de cada organización o a las situaciones que presenta la región; es una característica que se mantiene en las organizaciones campesinas abordadas en el estudio, las dos publican información original y mantienen interacción con otras publicaciones, inclusive de temas no inherentes al Catatumbo.

En ambas se logra "la convergencia, integración de múltiples formatos en una misma plataforma digital" (Nagel, 2012, p. 27), aclarando que normalmente es el texto el que prima, al ser este un formato indispensable en cada publicación realizada, brindando a los usuarios mayores elementos de comprensión del tema difundido. 


\section{Discusión y conclusiones}

Facebook como red social virtual creada en 2004 por Mark Elliot Zuckerberg, nació con la finalidad de conectar amigos en todo el mundo, y permitir a las personas construir una red donde se compartiera todo tipo de información en diversos formatos, lo anterior partiendo de su misión: "brinde a las personas el poder de construir una comunidad y acercar al mundo" (Facebook, 2021).

En ese sentido, Facebook no es considerado ni fue diseñado para ser un medio de comunicación masiva. Sin embargo, las organizaciones campesinas del Catatumbo, aunque son conscientes de esta realidad, aprovechan la herramienta para generar un patrón difusionista que visibilice sus acciones, transmitiendo sus discursos desde los propios protagonistas. Con lo anterior, las organizaciones campesinas logran impedir que las personas solo se queden con el discurso de las grandes empresas mediáticas, las cuales en algunas ocasiones desconocen las realidades del Catatumbo, porque no hacen presencia en el territorio.

Para Ascamcat y el Cisca, la red social virtual ofrece las posibilidades que antes no ofrecían con quienes tienen alianzas. Ahora es el equipo de comunicación de cada organización quien transmite la información sin intermediarios, convirtiéndose en una fuente fiel de las problemáticas, necesidades, exigencias y acciones emprendidas en la región, generando contenidos en diversos formatos y reduciendo costos de producción.

Adaptabilidad y flexibilidad son quizás dos elementos intrínsecos en las nuevas relaciones y sociedad participativa. Hoy es impensable concebir temas como democracia, cultura, Nación u otros, sin estas características que legitiman su permanencia en el tiempo. Recordando las palabras de Roland Colin, citado por Blanchet (2001) "la participación no es una receta, una técnica ni una metodología, sino cierto tipo de funcionamiento de las instituciones sociales y de la creación cultural que refleja un proyecto de sociedad y por consiguiente una opción política" (p. 177).

La llamada tecnopolítica, mencionada por Monterde, Rodríguez y Peña-López (2013), permite una ruptura con la cultura organizativa y comunicativa de la vieja izquierda. La cultura digital no opera de una forma esencialista entorno a unas figuras e ideas concretas e inmutables, sino en unos ámbitos de acción siempre de forma amplia y transversal, en las que la militancia y la participación ya no se definen bajo criterios de pertenencia ni identidad, sino entorno a problemas y necesidades concretas.

La libertad de información será un factor trascendental de los cambios en el nuevo orden de sociedad en red. Con mayores conocimientos y puntos de vista, los grupos podrán ser partícipes en la toma de decisiones antes limitadas a unos cuantos. Así, dice Castells (2009) que: 
Por tanto, una estructura social cuya infraestructura se base en redes digitales tiene las posibilidades de ser global. No obstante, la tecnología de redes y la organización en red son solo medios que reflejan las tendencias inscritas en la estructura social. (p. 51)

La antigua dicotomía entre realidad física y realidad virtual, es un obstáculo que debe superarse; ambas realidades deben ser un binomio y complementarse bidireccionalmente, porque así el impacto de las redes ciudadanas locales, regionales o nacionales tendrán visibilidad y capacidad internacional de transformación, política, económica, cultural o social. Es por esto por lo que Monterde, Rodríguez y Peña-López (2013) afirman que: "Ie|ntre la calle y la red no existe una relación dicotómica sino de simbiosis. El geoterritorio no puede entenderse sin el ciberterritorio, y viceversa" (p. 17).

Comunicar debe ser aptitud y actitud, "supone ponernos en disposición de comunicar; cultivar en nosotros la voluntad de entrar en comunicación con nuestros interlocutores" (Kaplún, 2002, p. 85). Por lo tanto, el deseo de un país diferente debe pasar por la visibilidad de nuevos temas en la agenda de los cibermedios. Hoy la capacidad ciudadana de informar en medios virtuales privilegia la banalidad, cientos de memes circulan por doquier, pero escazas iniciativas civiles de importancia son compartidas en red.

Para lograr un desarrollo trascendente ligado a un cambio tecnológico, es necesario la comprensión de la estrategia, definida por De Certeau (1970) como el "cálculo (o a la manipulación) de las relaciones de fuerzas que se hace posible desde que un sujeto de voluntad y de poder (una empresa, un ejército, una ciudad, una institución científica), resulta aislable" (p. 42), debemos relacionarla con la táctica, definida por el autor como el "cálculo que no puede contar con un lugar propio, ni por tanto con una frontera que distinga al otro como una totalidad visible. La táctica no tiene más lugar que el del otro" (De Certeau, 1990, p. L).

Millones de personas en el mundo respondemos casi sin pensarlo a las estrategias comerciales y gubernamentales de los medios, muchos de ellos no de servicio al público; "una región en la que los medios de gestión estatal funcionan como dispositivos de propaganda gubernamental" (Becerra, 2014, p. 65), es el escenario donde la táctica de sujetos creativos juega un rol decisivo en la reconfiguración de las colectividades como grupos no dominados sino dispuestos a trabajar con resistencia y propuestas su futuro, estos apoyados en medios digitales, tal es el caso de la Asociación de Campesinos del Catatumbo (Ascamcat) y el Comité de Integración Social del Catatumbo (Cisca).

Ambas organizaciones, a manera de ejemplo, resumen la capacidad del ser humano para organizarse, tejer redes sociales y formar capital social; sumado a ello la posibilidad de visibilizar y potencializar su actuación a instancias más allá de lo regional, logrando incidir en procesos de decisión que los afectan directa o indirectamente. 
Dichas organizaciones promueven su discurso contra hegemónico con ayuda de internet y las redes sociales, manifestando su postura en comunicados en sus Fan Pages oficiales de Facebook, Twitter, así como en páginas y blogs. Luego de doce años de funcionamiento, son claras las expresiones de un manejo consciente entre la estrategia impuesta y su táctica como respuesta.

\section{Referencias}

Ascamcat. (2018). Información de los seguidores de la Asociación Campesina del Catatumbo. [página de Facebook]. Facebook. Consultado el 26 de mayo de 2018. https://www.facebook. $\mathrm{com} /$ search/top/?q=ascamcat\%20-\%20oficial

Becerra, M. (2014). Medios de comunicación: América Latina a contramano. Nueva Sociedad, (249). https://nuso.org/articulo/medios-de-comunicacion-america-latina-a-contramano/

Blanchet, K. (2001). La gobernabilidad y sus críticos. El desarrollo participativo, entre deseos y realidades. Revista Internacional de Ciencias Sociales, (170). http://www.unesco.org/new/fileadmin/ MULTIMEDIA/HQ/SHS/pdf/170-fulltext170spa.pdf

Castells, M. (2009). Comunicación y poder. Alianza Editorial. https://www.felsemiotica.com/descargas/ Castells-Manuel-Comunicaci\%C3\%B3n-y-poder.pdf

Cisca. (2018). Información de los seguidores del Comité de Integración Social del Catatumbo Ipágina de Facebook]. Facebook. Consultado el 26 de mayo de 2018. https://www.facebook.com/cisca. comunicaciones/

Colombia TIC. (2018). Estadísticas Puntos Vive Digital en Norte de Santander. https://colombiatic.mintic. gov.co/679/w3-propertyvalue-36366.html

Duarte, F. y Pires, H., F. (2011). Inclusión digital, tres conceptos clave: conectividad, accesibilidad, comunicabilidad. https://revistes.ub.edu/index.php/aracne/article/view/26674

De Certeau, M. (1990). La invención de lo cotidiano. Universidad Iberoamericana Biblioteca Francisco Xavier Clavigero. https://monoskop. org/images/2/28/De_Certeau_Michel_La_invencion_de_ lo_cotidiano_1_Artes_de_hacer.pdf

De Ugarte, D. (2007). El poder de las redes. http://www.pensamientocritico.org/davuga0313.pdf

De Pablos C., J. M. (1999) Es que vamos hacia una sociedad de la información. Latina, Revista de Comunicación Social, (14). https://dialnet.unirioja.es/servlet/articulo?codigo $=1214182$

Facebook. (2021). Misión de la red social virtual Facebook. https://about.facebook.com/company-info/

Gómez M., C. (2015). Nuevas aproximaciones a la teoría de los usos sociales: Prioridades para comprender los procesos de apropiación de las TIC en sociedades diversas. Visiones interdisciplinarias de la diversidad cultural. Colección del Seminario de Investigación sobre sociedad del conocimiento y diversidad cultural de la UNAM. https://www.academia.edu/27589964/ Visiones_Interdisciplinarias_de_la_Diversidad_Cultural?auto $=$ download

Harto de Vera, F. (2006). Tipologías y modelos de democracia electrónica. Revista de Internet, Derecho y Política, 2. 
Hernández-Sampieri, R., Fernández-Collado, C. y Baptista-Lucio, P. (2014). Metodología de la Investigación. Interamericana Editores

Kaplún, M. (2002). Una pedagogía de la comunicación, el comunicador popular. Ediciones Ciespal. http:// www.aader.org.ar/admin/savefiles/352_Mario\%20Kaplun.pdf

Max-Neef, M. (1993). Desarrollo a escala humana. Nordan comunidad. http://www.max-neef.cl/ descargas/Max_Neef-Desarrollo_a_escala_humana.pdf

Mejía, C., G. (2016). Las organizaciones sociales que mueven el Catatumbo. Verdad Abierta. http:// www.verdadabierta.com/victimas-seccion/los-resistentes/6341-las-organizaciones-socialesque-mueven-el-catatumbo

Monterde, A., Rodríguez, A. y Peña-López, I. (2013). La reivindicación de la democracia en la sociedad red, neutralidad de la red, ética hacker, cultura digital, crisis institucional y nueva institucionalidad. Working Paper Series WP13, 004. Internet Interdisciplinary Institute. http:// tecnopolitica.net/sites/default/files/1774-6278-4-PB.pdf

Nagel, J. (2012). Principales barreras para la adopción de las TIC en la agricultura y en las áreas rurales. Comisión Económica para América Latina y el Caribe (Cepal). http://repositorio.cepal.org/ bitstream/handle/11362/4011/S2012079_es.pdf?sequence $=1$

Organización de las Naciones Unidas para la Alimentación y la Agricultura (FAO). (1994). Participación Campesina para una Agricultura Sostenible en Países de América Latina. http://www. fao.org/3/t3666s/t3666s04.htm\#: : text=Las\%20organizaciones\%20campesinas\%2C\%20 tambi\%C3\%A9n\%20llamadas,o\%20sociales\%20de\%20sus\%20miembros.

Paquienséguy, F. (2007). Las Tecnologías de la Información y la Comunicación y sus usos hoy. Revista Q, 1(2). https://repository.upb.edu.co/bitstream/handle/20.500.11912/6490/Las\%20 tecnolog\%C3\%ADas\%20de\%20informaci\%C3\%B3n\%20y\%20comunicaci\%C3\%B3n\%20y\%20 sus\%20usos.pdf?sequence $=1$ EisAllowed $=y$

Simon, J. P. (2016). La transformación digital, nuevos agentes en la industria de los medios y contenidos. Revista Telos, Cuadernos de Comunicación e Innovación. https://telos.fundaciontelefonica. com/archivo/numero 103/nuevos-agentes-en-la-industria-de-los-medios-y-contenidos/ 